Trauma Berufskrankh 2005 · 7[Suppl 1]:S69-S73 DOI 10.1007/s10039-004-0906-5

Online publiziert: 7. Juli 2004

() Springer-Verlag 2004

M. Jessel · H. Erhard · BG-Unfallkrankenhaus Hamburg

\title{
Mangelnde Abbildbarkeit berufsgenossenschaftlich relevanter Verletzungsarten
} Mögliche Auswirkungen

— Querschnittgelähmte,

- Schwerbrandverletzte,

- Polytraumatisierte,

- Osteitispatienten,

- Schwer-Schädel-Hirn-Verletzte,

- rekonstruktive Chirurgie,

- Replantationschirurgie sowie im

- rehabilitativen Bereich.

Alles sind Bereiche, die in ihrer Komplexität derzeit im DRG-System nur schlecht abgebildet sind und deren derzeitige Bewertungsrelationen großenteils keine Kosten deckenden Behandlungen erlauben.

\section{Erste Anpassungen nach der 2. Kalkulationsrunde}

In der AR-DRG-Version waren insgesamt 664 DRG kalkuliert. Im Rahmen der ersten deutschen Kalkulationsrunde 2003, in der nur die Teildaten der ersten Monate des Jahres 2002 vorlagen, wurden 642 DRG kalkuliert. Die Daten der 2. Kalkulationsrunde waren bereits deutlich valider und rekrutierten sich aus dem Gesamtjahreszeitraum 2002. Es konnten jetzt 824 DRG auf Grundlage der an der Kalkulationsrunde teilnehmenden Kliniken ermittelt werden, immerhin 176 DRG wurden neu kreiert, 16 DRG wurden gestrichen, und bei 126 DRG wurden Änderungen der Splitkriterien vorgenommen. Die selbst auferlegte Obergrenze von 800 DRG war bereits jetzt überschritten.

Während bei der ersten Kalkulationsrunde die Daten von 116 Krankenhäusern zur Verfügung standen, lieferten bei der 2 .
Kalkulationsrunde „immerhin“ 144 Krankenhäuser ( $8 \%$ der Leistungsdaten liefernden Krankenhäuser) Kostendaten, hierunter waren jetzt erstmalig 12 Universitätskliniken. Mit anderen Worten bedeutet das, dass das Gesundheitssystem in Deutschland derzeit auf Basis einer sehr dürftigen Datenlage von etwa $6 \%$ der deutschen Kliniken reformiert wird! In Kenntnis der erheblichen Aufwendungen für die teilnehmenden Kalkulationskliniken, eine funktionierende Kostenträgerrechnung aufzustellen, darf es nicht verwundern, wenn im Rahmen der nächsten Kalkulationsrunde, die die Gesamtjahresdaten des Jahres 2003 beinhaltet, noch immer keine validen Kostendaten deutscher Krankenhäuser vorliegen, mit denen das GDRG-System aufwandsgerecht umzusetzen ist.

\section{Kompressionseffekt mit Verzerrung der Erlössituation}

Bei Analyse der bisher in beiden Kalkulationsprojekten ermittelten Bewertungsrelationen fällt auf, dass es zu erheblichen Verschiebungen bisheriger Vergütungsmaßstäbe kommt. Begriffe wie Kompressionseffekt beschreiben, dass nach bisher festgelegten Bewertungsmaßstäben relativ einfache Krankenhausbehandlungen überproportional teuer und sehr aufwändige Behandlungen außerordentlich schlecht vergütet werden. Dies darf angesichts der schlechten Datenlage nicht verwundern.

- Tabelle 1 zeigt Beispiele für sehr teuer abgerechnete Fälle des Jahres 2003 mit den Änderungen 2004, 0 Tabelle 2 dagegen Bei- 
spiele für zu preisgünstig kalkulierte Fälle des Jahres 2003 mit den Änderungen 2004. Wo1A und B61A sind nicht kalkuliert.

Bereits jetzt, knapp 1 Jahr nach Beginn des Optionsjahrs, melden die Unfallkassen deutliche Mehrausgaben für den stationären Sektor, was nicht verwunderlich ist, wenn man sich die Bewertungsrelationen sowohl des Optionsjahres 2003 als auch die des Jahrs 2004 ansieht.

\section{Komplexbehandlungsvergütung}

Komplexbehandlungen sind, wie an den folgenden Beispielen dargestellt, extrem knapp kalkuliert (vergütet):

1. Für die erfolgreiche Replantation einer Hand nach Kreissägenamputationsverletzung wurde ein Handwerker im BG-Unfallkrankenhaus Hamburg 92 Tage statio- när behandelt - inklusive intensiver frührehabilitativer Maßnahmen. Im Ergebnis bestand nach 1 Jahr wieder Arbeitsfähigkeit als Handwerker. Als „Tageserlös“ für die 92 Tage akutstationärer Behandlung wäre dieser Fall bei einem bereits mit $2900 €$ großzügig angesetzten Basisfallwert mit $199 €$ ! (DRG Xo7Z) vergütet worden. Im Falle einer Stumpfbildung, die relativ einfach durchzuführen gewesen wäre und die man ohne Berücksichtigung weiterer prothetischer Versorgungen mit einer etwa 1wöchigen stationären Behandlung hätte „DRG-konform“ therapieren können, würde ein umgerechneter Tageserlös von etwa $376 €$ resultieren! Für den Fall der medizinisch und therapeutisch sehr aufwändigen Replantation würde das Krankenhaus unter den Bedingungen 2004 ein erhebliches finanzielles Risiko eingehen (evtl. Komplikationen mit weiteren nicht finanzierten

Tabelle 1

Sehr teuer abgerechnete Fälle

\begin{tabular}{|c|c|c|c|c|c|c|}
\hline \multirow[t]{2}{*}{ Eingriff bei/zur } & \multicolumn{2}{|l|}{ DRG } & \multicolumn{2}{|l|}{2003} & \multicolumn{2}{|l|}{2004} \\
\hline & 2003 & 2004 & $\begin{array}{l}\text { Relatives } \\
\text { Gewicht }\end{array}$ & $\begin{array}{l}\text { Erlös } \\
{[€]}\end{array}$ & $\begin{array}{l}\text { Relatives } \\
\text { Gewicht }\end{array}$ & $\begin{array}{l}\text { Erlös } \\
{[€]}\end{array}$ \\
\hline $\begin{array}{l}\text { Material- } \\
\text { entfernung }\end{array}$ & $123 Z$ & $123 Z$ & 0,732 & 1976 & 0,633 & 1709 \\
\hline Arthroskopie Knie & I18Z & I18Z & 0,854 & 2306 & 0,664 & 1793 \\
\hline $\begin{array}{l}\text { Außenknöchel- } \\
\text { fraktur }\end{array}$ & I13C & I13D & 1,326 & 3580 & 1,409 & 3804 \\
\hline $\begin{array}{l}\text { Distale } \\
\text { Radiusfraktur }\end{array}$ & I19Z & I18Z/I31Z & 1,0 & 2700 & $0,664 / 1,057$ & $1793 / 2854$ \\
\hline
\end{tabular}

Fälle des Jahres 2003 mit den Änderungen 2004, angenommene Baserate: $2700 €$

Kostensteigerungen) bei gleichzeitiger $\mathrm{Ge}$ wissheit, dass eine Kostendeckung in keinem Fall zu erreichen ist.

2. Eine junge Frau geriet bei einem KFZÜberschlag mit ihrem Arm zwischen Dach und Asphalt und zog sich hierbei schwerste Weichteilzerreißungen am Unterarm sowie multiple Frakturen an Unterarm und Hand zu. In mehrfach geplant durchgeführten Operationen im interdisziplinären Team mit Hand-, Plastischenund Unfallchirurgen konnte die Extremität bei sehr guter Funktion erhalten werden. Bei zeitgleich durchgeführten intensiven Rehabilitationsmaßnahmen wurde eine stationäre Verweildauer von 87 Tagen benötigt, die Minderung der Erwerbsfähigkeit auf Dauer betrug für die gesetzliche Unfallversicherung lediglich $20 \mathrm{v}$. H. Umgerechnet wären für diesen Fall $172 € /$ Tag vergütet worden. Auch in diesem Fall ist der selbstauferlegte Anspruch des BMGS „Das Geld folgt der Leistung“" nicht einmal im Ansatz erfüllt.

3. Ein Mann mit Verbrennungen von insgesamt $20 \% \mathrm{KOF}$ wurde 77 Tage stationär im BG-Unfallkrankenhaus Hamburg behandelt. Hiervon waren 12\% Verbrennungen Grad IIb-III am Körperstamm und 8\% Grad IIa an beiden Beinen. Die Beatmungszeit betrug $85 \mathrm{~h}$, die Behandlung auf der Schwerbrandverletztenintensivstation dauerte 2 Wochen. Chirurgisch wurden mehrfache Nekrektomien, Spalthauttransplantationen sowie Verbandwechsel in Narkose vorgenommen. Die zu erwartende MdE auf Dauer ist mit etwa 30-40 v. H.

Tabelle 2

Zu preisgünstig kalkulierte Fälle

\begin{tabular}{|c|c|c|c|c|c|c|}
\hline \multirow[t]{2}{*}{ Eingriff bei/zur } & \multicolumn{2}{|l|}{ DRG } & \multicolumn{2}{|l|}{2003} & \multicolumn{2}{|l|}{2004} \\
\hline & 2003 & 2004 & Relatives Gewicht & Erlös [€] & Relatives Gewicht & Erlös [€] \\
\hline $\begin{array}{l}\text { Polytrauma mit } \\
\text { Langzeitbeatmung }\end{array}$ & W01Z & $\begin{array}{l}\text { W01A-W01D/ } \\
\text { A06-A13 }\end{array}$ & 13,323 & 32.400 & $48,272-7,272$ & $130.334-20.862$ \\
\hline Polytrauma & W02Z & W02Z & 5,106 & 13.786 & 4,559 & 12.350 \\
\hline $\begin{array}{l}\text { Großflächige Verbren- } \\
\text { nung Grad II }\end{array}$ & Y02B & Y02B & 2,224 & 6005 & 3,694 & 9974 \\
\hline Replantation Hand & X03Z & X07Z & 1,735 & 4685 & 2,065 & 5575 \\
\hline Replantation Finger & $\mathrm{X} 02 \mathrm{Z}$ & X07Z & 1,368 & 3694 & 2,065 & 5575 \\
\hline Querschnitt & B61A & B61A & 4,351 & 11.784 & - & n.§6/KhEntG \\
\hline
\end{tabular}

Fälle des Jahres 2003 mit den Änderungen 2004, W01A und B61A nicht kalkuliert, angenommene Baserate: $2700 €$ 
anzunehmen. Die abzurechnende DRG für diesen Fall ist Yo2A mit einem Relativgewicht von 5,798 bei einer kalkulierten mittleren Verweildauer von 25 Tagen. Ab Überschreiten der oberen Grenzverweildauer von 42 Tagen wird mit jedem Tag ein Zuschlag fällig, sodass schließlich ein Effektivgewicht von 10,313 resultiert. Bei einer wiederum großzügigen Veranschlagung des Basisfallwerts von $2900 €$ resultiert ein „Tageserlös“ von $388 €$. An dieser Stelle sei angemerkt, dass der bisher gültige Tagespflegesatz für die Schwerbrandverletztenintensivstation derzeit $3913 €$ beträgt, bedingt durch die erheblichen Kosten der Vorhalteleistungen.

Diese 3 Beispiele legen die derzeitige mangelnde Abbildbarkeit von Komplexverletzten im DRG-System offen.

Dies gilt in gleicher Weise für Querschnittgelähmte, die das BG-Unfallkrankenhaus Hamburg in der überwiegenden Zahl der Fälle als Selbstversorger in die häusliche Umgebung verlassen. Im Optionsjahr 2003 waren nur 27,5 Tage für die akutstationäre Behandlung Querschnittgelähmter zugebilligt. Äußerst wichtig ist aber gerade hier eine in die Akutbehandlung integrierte intensive Frührehabilitationsbehandlung. Der Versicherte soll so gut rehabilitiert wie irgend möglich in die häusliche Umgebung entlassen werden. Dass mit dieser kurzen Zeitdauer serienweise Entlassungen in Pflegeeinrichtungen produziert worden wären, liegt auf der Hand.

\section{Herausnahme einiger Verletzungen aus DRG-Kalkulation}

Die Einbindung externen Sachverstands in Form zahlreicher Einwände der Fachverbände hat zumindest teilweise Wirkung gezeigt, so hat das BMGS die Querschnittgelähmten, sofern sie sich in der DRG B61A/B wiederfinden, zunächst aus der DRG-Kalkulation herausgenommen.

Diese Forderung steht noch für einige andere Bereiche in der Traumatologie. So sind z. B. Forderungen zur Herausnahme der Komplexverletzungen, der Replantationen und Polytraumen durchaus nicht unbegründet. Für Teilbereiche der Verbrennungsmedizin ist eine temporäre $\mathrm{He}$ rausnahme bereits beschlossen, was die DRG Yo1Z und Y61Z betrifft. Hierbei handelt es sich allerdings nicht um eine prinzipielle Herausnahme aus dem System,

Trauma Berufskrankh 2005 $\cdot 7$ [Suppl 1]:S69-S73

DOI 10.1007/s10039-004-0906-5

(c) Springer-Verlag 2004

\section{Jessel $\cdot H$. Erhard}

\section{Mangelnde Abbildbarkeit berufsgenossenschaftlich relevanter Verletzungsarten. Mögliche Auswirkungen}

\section{Zusammenfassung}

Mit dem neuen Fallpauschalensystem werden die Diagnosis related groups als Vergütungsgruppen nicht nur für den Bereich der gesetzlichen Krankenversicherung, sondern auch für weite Bereiche der gesetzlichen Unfallversicherung eingeführt. Der Gesetzgeber bezieht die Träger der gesetzlichen Unfallversicherung mit ein, obgleich die Aufgabenstellungen zwischen beiden Systemen variieren und sich die Interessen beider Versicherungssysteme grundlegend unterscheiden. Es wird dargelegt, dass die Einführung des DRG-Systems für die gesetzliche Unfallversicherung unter derzeitigen Prämissen keineswegs einen Qualitätsgewinn darstellt und hierdurch sogar Kostensteigerungen drohen.

\section{Schlüsselwörter}

Fallpauschalensystem · Diagnosis related groups · Gesetzliche Krankenversicherung · Gesetzliche Unfallversicherung

\section{Impossibility of coding injury types relevant to the work of employers' liability insurance associations. Possible ramifications}

tems are fundamentally different. This paper presents the thesis that the introduction of the DRG system is not a way of improving quality in the present situation and that it even threatens to increase costs.

\section{Keywords}

Per-case payment - Diagnosis-related groups - Legally required health insurance. Legally required accident insurance 
Tabelle 3

Kostenanalyse für gesetzliche Unfallversicherung

\begin{tabular}{|c|c|c|}
\hline Maßnahme & $\begin{array}{l}\text { Extremitätenerhalt } \\
\text { Unterschenkel } \\
\text { Kosten }[€]\end{array}$ & $\begin{array}{l}\text { Amputation } \\
\text { Unterschenkel } \\
\text { Kosten [€] }\end{array}$ \\
\hline Stationäre Behandlung & 28.387 & 11.000 \\
\hline Prothesenversorgung & & 65.000 \\
\hline $\begin{array}{l}\text { Hilfsmittelversorgung } \\
\text { (orthopädische Schuhe, Gehapparat) }\end{array}$ & 28.500 & \\
\hline Verletztengeld & 22.500 & 15.000 \\
\hline Rentenleistungen & 328.000 & 442.000 \\
\hline Umschulung/Übergangsgeld & & 107.000 \\
\hline Gesamtkosten GUV & $\sim 407.00$ & 661.000 \\
\hline
\end{tabular}

sondern nur um eine zeitweise „Zurückstellung", da die bisher ermittelten Kostendaten eine Pauschalierung innerhalb des DRG-Systems bisher nicht zuließen.

Prinzipiell ist eine DRG-Vergütung auch der oben genannten Problembereiche sicherlich möglich, hierfür bedarf es aber mehrjähriger vollständiger Datenerhebungen zur korrekten Kostengewichtsermittlung.

\section{Unterschiedliche Konzepte - eine Vergütung?}

Die Struktur der DRG ist auf die gesetzliche Krankenversicherung abgestimmt. Einer der wesentlichen Eckdaten der DRGKalkulation ist die kalkulatorisch ermittelte mittlere Verweildauer, wobei bis zum Erreichen der oberen Grenzverweildauer keine Mehrerlöse vergütet werden. Die sich hieraus ableitenden Direktiven sind klar: möglichst zügige stationäre Entlassung zur Reduzierung der Kosten im akutstationären Bereich. Eine Kontrolle der Kostenentwicklung im nachgeordneten medizinischen und sozialen Rehabilitationsbereich erfolgt nicht, ebenfalls keine Kontrolle späterer Kostengestaltungen im Rentenwesen. Die DRG sind abgestimmt auf das duale Vergütungssystem, wobei etwa ein Kostenanteil von 10\% für den Investitionsbereich nicht über die DRG, sondern über die öffentliche Hand vergütet wird.

Vom BMGS ist der Anwendungsbereich über den SGB-V-Bereich hinaus ausgedehnt worden auf weite Teilbereiche des SGB VII. So sind Patienten der gesetzlichen Unfallversicherung, die in Kran- kenhäusern behandelt werden, deren Träger nicht die gesetzliche Unfallversicherung ist, prinzipiell nach DRG abzurechnen. Das Fallpauschalengesetz findet ebenfalls Anwendung auf Patienten, die zu Lasten der Krankenversicherung in Kliniken der gesetzlichen Unfallversicherung liegen. Vom Gesetzgeber werden hier $2 \mathrm{Be}$ reiche mit gleichen Vergütungsformen abgegolten, deren Aufgabenstellungen deutlich differieren:

Auf der einen Seite die krankenversicherten Patienten, deren Leistungen wirtschaftlich und nur im notwendigen Umfang erbracht werden dürfen, auf der anderen Seite die gesetzlich Unfallversicherten, deren Leistungen mit allen geeigneten Mitteln zu erfolgen haben. Während bei der gesetzlichen Krankenversicherung in erster Linie die momentan anstehende Akutbehandlung im Vordergrund des Interesses steht, ist bei der Behandlung nach SGB VII stets die Gesamtbehandlung von Interesse: vom Unfall bis zur Wiedereingliederung in das Berufsleben inklusive aller medizinischen und sozialgesellschaftlichen Rehabilitationsmaßnahmen, einschließlich etwaiger Rentenleistungen. Die unterschiedlichen Interessen liegen auf der Hand: Bei der gesetzlichen Krankenversicherung heißt das Kostenreduktion vornehmlich durch Kostensenkung im Rahmen der Akutbehandlung. Bei der gesetzlichen Unfallversicherung heißt das schnellstmögliche Rückkehr an den Arbeitsplatz unter zur Verfügungstellung aller erforderlichen Mittel. Das Interesse des Unfallversicherungsträgers ist ein bestmögliches Ergebnis zur Vermeidung späterer
Rentenleistungen. Der Ausgabenschwerpunkt liegt hier u. a. auf dem Rehabilitations- und Rentenbereich (• Abb. 1).

Für die gesetzliche Unfallversicherung sei noch einmal dargelegt, dass das Fallpauschalengesetz in seiner jetzigen Form qualitätsfördernden Aspekten eher zuwider läuft, als diese zu fördern. Die Favorisierung der auf das akute Geschehen eingeschränkten (kurzsichtigen!) Sichtweise gegenüber einer gesamtumfassenden Fürsorge vom Unfall bis zur Wiederarbeitsfähigkeit bzw. Rentenleistung ist für die gesetzliche Unfallversicherung qualitätsmindernd und eher Kosten treibend.

An 2 Beispielen sei dies verdeutlicht: 1. Die Replantation einer Hand bei einem Handwerker (s. Beispiel 1) verursachte Kosten von etwa $29.000 €$ für die Akutbehandlung bei einer stationären Verweildauer von 92 Tagen. Die Berechnungen der Folgekosten mit Verletztengeldzahlungen und Rentenbezügen, hochgerechnet auf das 80 . Lebensjahr, beliefen sich auf insgesamt etwa $396.000 €$.

Im Vergleich hierzu ergeben die Berechnungen für den angenommenen Fall der Stumpfbildung bei vergleichsweise niedrigen Primärkosten der Akutbehandlung von etwa $15.900 €$ (inklusive Prothesenanpassung) deutlich höhere Gesamtkosten von etwa $654.000 €$ für die GUV. Hier eingerechnet wurde die stationäre Behandlung inklusive Rehabilitationstherapie und Versorgung mit Prothese. Ebenfalls wurden Kosten für Verletztengeldzahlungen, Umschulungsmaßnahmen, Rentenleistungen sowie für Prothesenerneuerungen und Reparaturen eingerechnet.

2. Einem jungen Mechaniker drohte bei seit 13 Jahren unbeherrschter Unterschenkelosteitis nach Tibiamarknagelung nach ehemals offener Unterschenkelfraktur eine Unterschenkelamputation. Nach Vorstellung im BG-Unfallkrankenhaus Hamburg gelang nach insgesamt 3 stationären Aufenthalten die Infektberuhigung mit Segmentresektion und schließlich Stabilitätswiederherstellung durch langstreckigen Wiederaufbau der Tibia. Während vorher eine MdE von $40 \%$ zuerkannt war, Berufsunfähigkeit und Unterschenkelamputation drohten, war die MdE jetzt mit $30 \%$ bewertet, und die Tätigkeit als Mechaniker konnte weiterhin ausgeübt werden. 


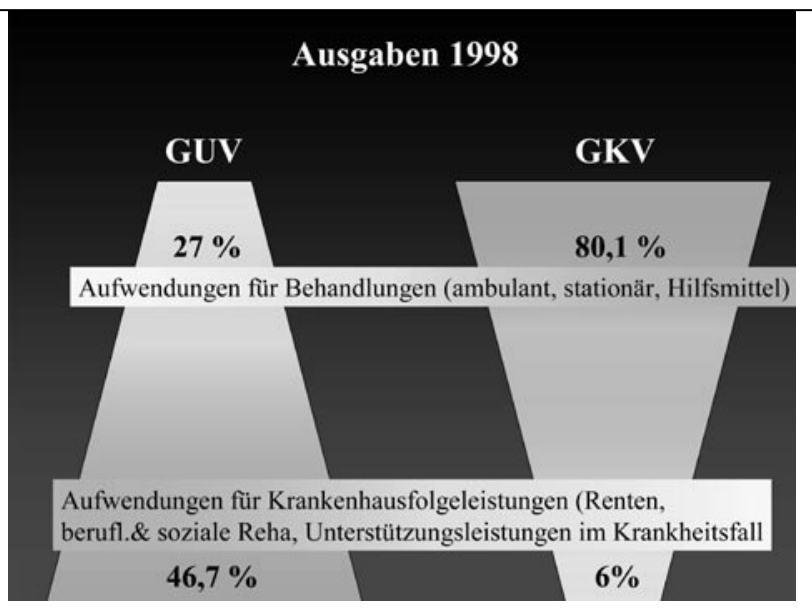

Abb. $1 \Delta$ Vergleich der Ausgabesituation bei gesetzlicher Unfallund Krankenversicherung

Die Kostenanalyse (hochgerechnet auf das 8o. Lebensjahr) ergibt für den Fall des Unterschenkelerhalts relativ hohe stationäre Akutbehandlungskosten, allerdings im Vergleich zum Amputationsfall deutlich geringere Folgekosten, wie in • Tabelle 3 dargestellt.

Unter derzeitigen DRG-Bedingungen würden sich für den geschilderten Fall im BG-Unfallkrankenhaus Hamburg gegenüber der Entgeltregelung nach Pflegesätzen bei den 3 akutstationären Behandlungen Mindereinnahmen für das Krankenhaus von jeweils $25 \%$, 33\% und $40 \%$ ergeben. Im Amputationsfall wäre für die Klinik das Risiko einer ausbleibenden Kostendeckung deutlich geringer, wenn die weitere prothetische Versorgung und gehschulerische Weiterbehandlung ambulant fortgeführt werden würden. Dass hierdurch bei wahrscheinlich verlängerter Prothesenanpassungs- und -versorgungszeit und dadurch verlängerter Heilbehandlung und Arbeitsunfähigkeit bei augenscheinlich primär günstigerer Versorgung gesamt gesehen höhere Ausgaben resultieren, ist zu befürchten.

An diesen Beispielen zeigt sich sehr anschaulich, dass trotz höheren primären akutstationären Behandlungskosten insgesamt gesehen bei bestimmten Komplexverletzungen die Gesamtkosten gegenüber der augenscheinlich primär kostengünstigeren Behandlung deutlich geringer sein können, diese werden allerdings aus DRG-Perspektive nicht berücksichtigt. Dies mag daran liegen, dass die Krankenkassen für die Folgekosten (Umschulungsmaßnahmen, Rehabilitationsleistungen,
Rentenleistungen) in der Regel nicht aufkommen und dass das DRG-Reglement auf das SGB V abgestimmt ist. Dass dies nicht das Interesse der Kostenträger der gesetzlichen Unfallversicherung sein kann, liegt auf der Hand.

\section{Korrespondierender Autor}

\section{Dr. M. Jessel}

BG-Unfallkrankenhaus Hamburg, Bergedorfer Straße 10, 21033 Hamburg

E-Mail: M.Jessel@buk-hamburg.de

Interessenkonflikt: Der korrespondierende Autor versichert, dass keine Verbindungen mit einer Firma, deren Produkt in dem Artikel genannt ist, oder einer Firma, die ein Konkurrenzprodukt vertreibt, bestehen. 